\title{
Application of polarimetry to test the models of thermonuclear plasma and determine the safety factor profile
}

\author{
Bohdan Bieg, Janusz Chrzanowski, Yury A. Kravtsov \\ Maritime University Szczecin, Waty Chrobrego 1-2, 70-500 Szczecin
}

Received August 30, 2015; accepted September 29, 2015; published September 30, 2015

\begin{abstract}
On the basis of the angle variables technique (AVT), changes in the polarimetry state of an electromagnetic wave passing through thermonuclear plasma have been analysed. Numerical calculations have been carried out for typical profiles of current density flowing through plasma. Then, for a given current density model the safety factor profile has been designated. All numerical calculations have been performed for plasma parameters that occur in real reactors.
\end{abstract}

The ITER project is the next phase in the development of tokamak technology. There are many challenging diagnostic problems related to both plasma instabilities and performance optimization of the plasma that need to be solved in order for ITER to meet its demanding performance objectives.

Due to the fact that ITER will be only an intermediate stage on the way to construct an industrial thermonuclear reactor, its diagnostic systems must additionally provide measurements to help understand plasma behavior and optimize fusion performance. Because of the extreme environment inside the tokamak vacuum vessel, these systems must cope with a range of conditions and phenomena not previously encountered by diagnostic technology, while performing with great accuracy and precision. Therefore it is clear that diagnostics of the industrial thermonuclear reactors will generally be based on elaborated models that allow to obtain maximum information despite a limited number of diagnostic tools.

In this paper the changes of the polarization state of an electromagnetic wave are examined for typical models of current density flowing through plasma. The paper is structured as follows. A short description is presented of the current flowing through plasma in the context of a safety factor profile $q$. The basics equations of the angular variable techniques (AVT) introduced by the authors are also briefly presented. Next are shown numerical calculation of the changes of polarization state for different density current profiles and their corresponding safety factor profile.

An important physical quantity related to the control of nonhomogeneous transport is the safety factor profile ( $q$-profile). The $q$-profile is a common heuristic for setting operating conditions that avoid undesired MHD instabilities. Additionally, recent studies have shown the importance of the $q$-profile in triggering internal

*E-mail: b.bieg@am.szczecin.pl transport, which significantly improves the energy confinement and assists in generating sawteeth that allow the removal of fusion ash from the central plasma.

The safety factor $q$ that describes the ratio of poloidal to toroidal rotations of the magnetic field line on the magnetic surface is defined as

$$
q(r)=\frac{1}{2 \pi} \oint \frac{1}{R} \frac{B_{\varphi}}{B_{\theta}} \mathrm{d} s
$$

where the integral is taken on the flux surface where the magnetic field lines lie. In this equation $r$ is the minor radius of the flux surface, $R$ the major radius and $B_{\varphi}, B_{\theta}$ mean the toroidal and poloidal magnetic fields respectively (Fig. 1). For a large aspect-ratio tokamak of a circular cross section the equations takes form

$$
q(r)=\frac{r B_{\phi}}{R_{0} B_{\theta}}
$$

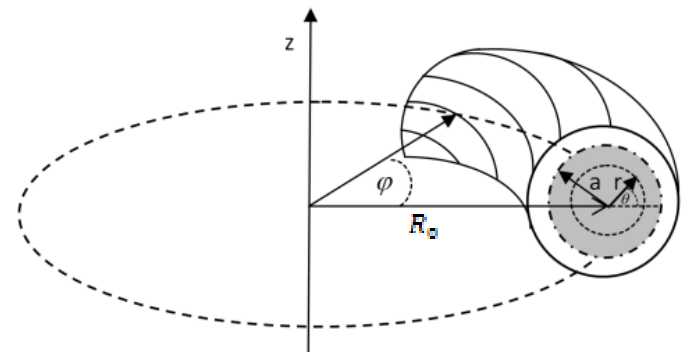

Fig. 1. The tokamak geometry.

This means that the key to determine the profile of $q$ is the knowledge of poloidal magnetic field $B_{\theta}$ produced by current flowing through the plasma. Therefore it is convenient to use the parameterization of the current profile for which $q(r)$ can be determined analytically. A suitable class of current profiles with this property is given by

$$
j(r)=j_{0}\left(1-\left(\frac{r}{a}\right)^{2}\right)^{v}
$$

where the current profile is parameterized by $v$ ( $a$ is the mean effective minor radius of the last closed flux 
surface: Fig. 1). In this case we can obtain the radial profile of the current enclosed by the surface with radius $r$

$$
I(r)=j_{0} \frac{\pi a^{2}}{\mu+1}\left(1-\left(1-\left(\frac{r}{a}\right)^{2}\right)^{v+1}\right)
$$

Consequently, the corresponding poloidal magnetic field $B_{\theta}(r)$ can be calculated directly from Ampere's equation

$$
\begin{gathered}
B_{\theta}(r)=\frac{\mu_{0} I(r)}{2 \pi r} \\
=\frac{\mu_{0} j_{0}}{2 r} \frac{a^{2}}{v+1}\left(1-\left(1-\left(\frac{r}{a}\right)^{2}\right)^{v+1}\right)
\end{gathered}
$$

what substituting into Eq. (2) gives the profile $(r)$

$$
\mathrm{q}(\rho)=\frac{2(\nu+1) \rho^{2} \mathrm{~B}_{\varphi}}{R_{0} \mu_{0} \mathrm{j}_{0}\left(1-\left(1-\rho^{2}\right)^{v+1}\right)}
$$

where $\rho=r / a$ means normalized radius.

The polarization state of an electromagnetic wave could be characterized by azimuthal $-\psi$ and ellipticity $-\chi$ angles. The evolution of these parameters is described by the equations of angular variables technique (AVT) [4]:

$$
\begin{gathered}
\frac{\mathrm{d} \psi}{\mathrm{d} \sigma}=\frac{1}{2}\left(\Omega_{3}-\left(\Omega_{1} \cos (2 \psi)\right.\right. \\
\left.\left.\quad+\Omega_{2} \sin (2 \psi)\right) \tan (2 \chi)\right), \\
\frac{\mathrm{d} \chi}{\mathrm{d} \sigma}=\frac{1}{2}\left(\Omega_{1} \sin (2 \psi)-\Omega_{2} \cos (2 \psi)\right)
\end{gathered}
$$

here $\sigma$ is the length along the ray and $\Omega_{1,2,3}$ are plasma parameters, traditionally used in plasma polarimetry [5], coefficients $\Omega_{1,2}$ corresponds to Cotton-Mouton effects, and coefficient $\Omega_{3}$ - to Faraday phenomenon [1,3,5]:

$$
\begin{gathered}
\Omega_{1}=C_{1} \lambda^{3}\left(B_{x}^{2}-B_{y}^{2}\right) N_{e} ; \\
\Omega_{2}=C_{2} \lambda^{3}\left(2 B_{x} B_{y}\right) N_{e} ; \\
\Omega_{3}=C_{3} \lambda^{2} B_{z} N_{e}
\end{gathered}
$$

within the SI system the constants $C_{i}$ are: $C_{1}=C_{2}=$ $2.45681 \cdot 10^{-11} \mathrm{~m}^{-1} \mathrm{~T}^{-2} ; C_{3}=5.26241 \cdot 10^{-13} \mathrm{~T}^{-1}$.

In order to make a numerical calculation, a simple model of plasma configuration has been chosen: plasma with a circular cross-section of the magnetic flux surfaces, parabolic density profile $N_{e}=N_{0}\left(1-\rho^{2}\right)$ and toroidal current density distribution given by Eq. (3).

The value of $j_{0}$, was chosen to obtain a definite value for the total plasma current $I_{0}$. The magnetic field components, at the point with the radius $R$ and normalized radius $\rho$, are [2]:

$$
B_{\varphi}=B_{0} \frac{R_{0}}{R}, \quad B_{\theta}=\frac{\mu_{0} I_{0}}{2 \pi a} \frac{1-\left(1-\rho^{2}\right)^{v+1}}{\rho}
$$

where $B_{0}$ is the toroidal magnetic field on the magnetic axis of the plasma with the major radius $R_{0}$. For such cords the magnetic field components in the beam reference frame are: $B_{x}=B_{\varphi}, B_{y}=B_{\theta} \sin \theta$ and $B_{z}=$ $B_{\theta} \cos \theta$.

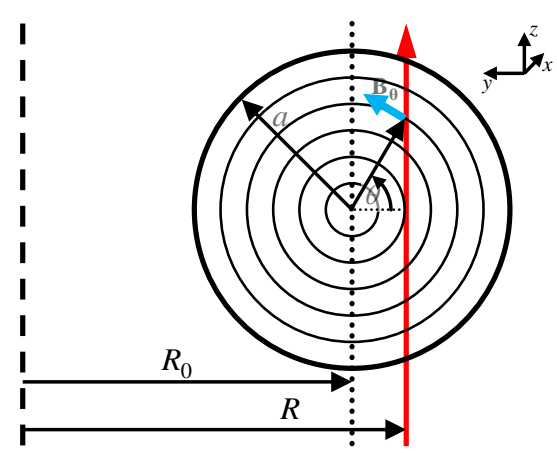

Fig. 3. Probing beam directed parallel to the main axis of the tokamak.

Numerical calculations were done for electromagnetic wave $\lambda=195 \mu \mathrm{m}$ and input polarization $\psi_{0}=\pi / 4$ and $\chi_{0}=0$, for a polarimeter featuring a set of parallel vertical probing beams was launched from the bottom of the plasma towards the top of the plasma (Fig. 3). Similar plasma parameters have been chosen to those of the large thermonuclear plasma devices: $R_{0}=3 \mathrm{~m}, \quad R=4 \mathrm{~m}$, $N_{0}=0.75 \cdot 10^{20} \mathrm{~m}^{-3}, B_{0}=4.8 \mathrm{~T}, I_{0}=3 \mathrm{MA}$.

Treating them as fixed, we examined changes in the polarization of the test beam for different values of the parameters $v$ and $a$. Then we examined their impact on the profile of a safety factor. The results of the numerical calculations are shown in the graphs below.

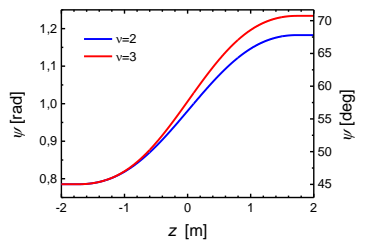

Fig. 4. Changes of the azimuthal angle $\psi$ along the path for two different values $v$ for $a=2 \mathrm{~m}$.

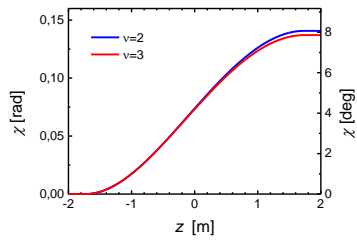

Fig. 5. Changes of the ellipticity angle $\chi$ along the path for two different values $v$ for $a=2 \mathrm{~m}$.
The graphs above show that the changes of Faraday rotation for the beam passing through tokamak plasma are so big that they can be observed with the simplest polarimetric systems (Fig. 4). But more important is the fact that they allow to detect the difference of the azimuthal angle even in the case of minor changes in the parameter $v$ so important for determining the current density distribution flowing through the plasma (Fig. 6). 
We observe practically a linear increase of the azimuthal angle with increasing of the parameter $v$, wherein the unit change of parameter $v$ corresponds to the change of the azimuth angle $\Delta \psi \approx 3^{0}$.

Changes in ellipticity angle $\chi$ related to the CottonMouton effect of the same test beam passing through the plasma are also significant and easy to detect (Fig. 5). However, in this case, the changes of the ellipticity angle are less sensitive to the changes in the parameter $v$ (Fig. $7)$. The increase in the parameter $v$ leads to a decrease in the angle of ellipticity $\chi$ - the unit change of the parameter $v$ corresponds to the change in the ellipticity angle in a range of 0.2 degree.

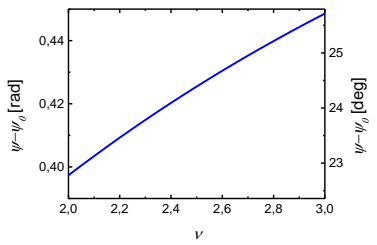

Fig. 6. Changes of the azimutha angle $\psi$ in dependency on $v$.

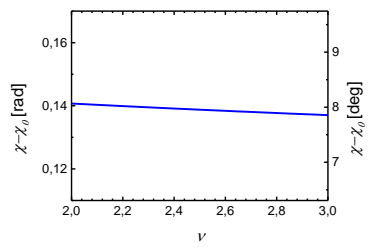

Fig. 7. Changes of the ellipticity angle $\chi$ in dependency on $v$.
A further important parameter in determining the distribution of the current flowing through the plasma is the minor radius $a$. In Figures $8-9$ are presented the results of numerical calculations showing the effect of the cross-section size of the plasma for a change of the azimuth and ellipticity angles.

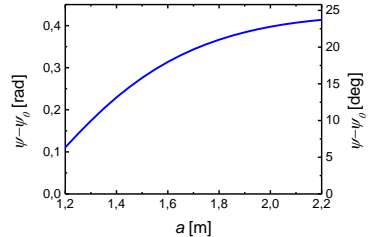

Fig. 8. Changes of the azimuthal angle $\psi$ in dependency on the minor radius $a$ for $v=2$.

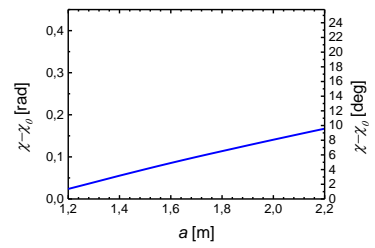

Fig. 9. Changes of the ellipticity angle $\chi$ in dependency on the minor radius $a$ for $v=2$.
As follows from the graphs above, the changes in the cross-section of the plasma have a very substantial impact on Faraday rotation (Fig. 8). Also explicit is the impact of minor radius $a$ on the change of the ellipticity angle $\chi$, where the unit change of $a$ corresponds to the change $\Delta \chi \approx 8^{\circ}$.

Using Eq. (6), we can determine the profile of the safety factor $q(\rho)$ which is so important for the stability of the reactor. Below on the graphs 10 and 11 are shown distributions of the profile $q(\rho)$ for two different values of the minor radius $(a=1.6 \mathrm{~m}$ and $a=2 \mathrm{~m})$ for $v=$ 2.4, 2.6, 2.8 and 3.0, whereas the other parameters are fixed.

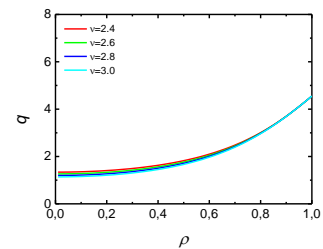

Fig. 10. Profile $q(\rho)$ for $a=$ $1.6 \mathrm{~m}$.

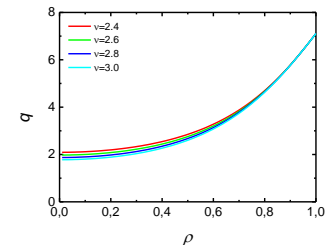

Fig. 11. Profile $q(\rho)$ for $a=2 \mathrm{~m}$
The analysis of the graphs above shows how significant for the value of a safety factor at the edge is the minor radius of the tokamak. It is also worth noting that increasing the parameter $v$ leads to decreasing the value of a safety factor at the central point.

Performed numerical calculations confirm that polarimetry may be an important component of comprehensive thermonuclear plasma diagnostics. Originally, one of the constraints was the structure of the differential Eqs. (12), which does not allow for direct reconstruction of the plasma parameters through polarimetric measurements in a general situation, in which there is strong coupling between the Faraday effect and that of Cotton-Mouton. However, suitable methods of inversion [4-6] allow basically to bypass this difficulty making it possible to use all potential channels for polarimetric measurements. In this way, even by simplified models of plasma we can determine, in real time, plasma parameters of our interest by measuring changes in the polarization of the electromagnetic wave. In the present article we concentrated on presenting the possibility of using polarimetric measurements to analyze the current flowing through the plasma and the poloidal magnetic field produced by this current.

It turns out that even for conditions such as those that exist in currently working tokamaks, the polarimetric methods are so sensitive [7] that they can detect even subtle changes of parameters that determine the density of the current flowing through the plasma. Consequently, the knowledge of parameters, such as $a, v$ allows to reconstruct the profile $q(\rho)$ which is so important from the perspective of the reactor stability

\section{References}

[1] B. Bieg, J. Chrzanowski, Yu.A. Kravtsov, A. Murari, F. Orsitto, Nucl. Instr. Meth. Phys. Res. Sect. A 720, 157 (2013).

[2] J. Wesson, Tokamaks (Clarendon Press, Oxford 2004).

[3] S.E. Segre, J. Opt. Soc. Am. A 18, 2601 (2001).

[4] Yu.A. Kravtsov, J. Chrzanowski, D. Mazon, Fus. Eng. Des. 86, 1163 (2011).

[5] Yu.A. Kravtsov, J. Chrzanowski, D. Mazon, Eur. Phys. J. D, 63, 135 (2011).

[6] J. Chrzanowski, Yu.A. Kravtsov, D. Mazon, Nukl. 57, 37 (2012).

[7] A.J.H. Donné, Plasma and Fusion Research, 8, 2102084 (2013). 